\title{
Nigritae / Nige(i)r, Nigris / Ge(i)r, Gir, Giris... (Nom de fleuve)
}

Salem Chaker

\section{OpenEdition}

12 Journals

Édition électronique

URL : https://journals.openedition.org/encyclopedieberbere/2743

DOI : 10.4000/encyclopedieberbere. 2743

ISSN : 2262-7197

Éditeur

Peeters Publishers

\section{Édition imprimée}

Date de publication : 5 octobre 2012

Pagination : 5556-5558

ISBN : 978-90-429-2718-6

ISSN : 1015-7344

Référence électronique

Salem Chaker, « Nigritae / Nige(i)r, Nigris / Ge(i)r, Gir, Giris... (Nom de fleuve) », Encyclopédie berbère [En ligne], 34 | 2012, document N58b, mis en ligne le 15 décembre 2020, consulté le 17 février 2022. URL : http://journals.openedition.org/encyclopedieberbere/2743 ; DOI : https://doi.org/10.4000/ encyclopedieberbere. 2743

Ce document a été généré automatiquement le 17 février 2022.

(C) Tous droits réservés 


\title{
Nigritae / Nige(i)r, Nigris / Ge(i)r, Gir, Giris... (Nom de fleuve)
}

\author{
Salem Chaker
}

1 De la notice précédente, il ressort que pour les spécialistes de géographie antique, sur la base de l'analyse systématique des diverses sources, la tribu des Nigritae tire son nom d'un hydronyme qui présente des formes assez différenciées : Nigeir, Nigris, Gir, Geir, Giris..., mais qui semblent renvoyer toutes à un même fleuve, approximativement localisé dans l'actuel Maroc atlantique méridional et souvent assimilé aux mythiques sources occidentales du Nil par les auteurs antiques.

2 La difficulté, pour le berbérisant, réside dans l'alternance entre une forme à initiale nasale /n/ et une forme sans cette nasale, qui est assez troublante car, dans la morphologie du nom berbère, une telle alternance initiale est improbable: elle ne correspond à aucun phénomène préfixai régulier connu. On serait a priori en droit de considérer ces deux formes comme étrangères l'une à l'autre.

Néanmoins, puisque les spécialistes de l'antiquité considèrent comme acquis que ces deux noms désignent bien un même et seul fleuve, l'hypothèse d'un lien entre les deux formes peut/doit être explorée malgré tout. Sur la base des données berbères, on peut envisager la piste explicative suivante : le doublet (à nasale initiale/sans nasale initiale) pourrait en fait relever d'une mauvaise coupe syntaxique faite par les auteurs anciens, la forme à nasale initiale $/ \mathrm{n} /$ étant un syntagme nominal :

Préposition $N(« \mathrm{de} »)+$ Nom $=n+G(e) i r=« \mathrm{de} / \mathrm{du}$ [fleuve] G(e)ir ».

Hypothèse qui permettrait d'ailleurs d'expliquer comment l'on est passé du nom d'un fleuve à celui d'une population : [gens] $N($ de) G(e)ir. Si cette hypothèse est fondée, le nom indigène du fleuve était donc: Geir/Gir... On notera que la préposition ", « de/du », introduisant un complément nominal est attestée, avec cette fonction, dès l'époque libyque, comme le montre RIL 1 (L 6 \& 7) - elle date au moins du II ${ }^{\mathrm{e}}$ siècle avant J.C. qui donne...N ZLH, «[ouvriers] du fer »,...N SYR, [ouvriers] du bois », syntagmes nominaux déterminatifs de structure et de forme quasiment berbères.

5 Or, cet hydronyme Geir/Gir a au moins un pendant dans l'hydronymie nord-africaine contemporaine : l'oued Guir, dans la région de Béchar (Sahara algérien). 
Mais aussi dans le lexique berbère : la racine GR est bien attestée et même tout à fait pan-berbère, donc ancienne, avec des signifiés divers : «lancer, jeter, rejeter, produire, introduire, se précipiter, débouler, s'élancer, jaillir...». En touareg (Foucauld, Dictionnaire touareg-français, 1950, I, p. 472), le nom féminin têgert signifie : « ruisseau, filet d'eau (permanent ou à peu près permanent)»; sous forme redoublée à valeur expressive, GRGR, elle signifie " couler à flots » (kabyle ; sur la racine GR et GRGR, cf. K. Naït-Zerrad, Dictionnaire des racines berbères, III, Louvain/Paris, Peeters, 2002, p. 848 et suivantes). Il y a donc une réelle compatibilité sémantique avec la dénomination d'un cours d'eau.

7 On peut imaginer que les Nigritae étaient les habitants d'une région où coulait un fleuve a débit important, ne serait-ce que de manière saisonnière ou par intermittence, dont le nom était fondé sur la racine $G R$.

8 Toujours à partir de cette même racine GR, une autre possibilité d'explication, serait de relier les deux formes antiques au doublet attesté dans le domaine chleuh (qui correspond d'ailleurs à la région où pouvait se trouver le Nigris/Gir et les Nigritae) : ger/gir/gar et nger/inger, "entre (deux), parmi, au milieu de, au centre de...", qui connaît une forme secondaire expressive à redoublement en touareg: gérégéré "moyen» (Cf. Naït-Zerrad, op. cit., p. 851-852). En berbère actuel, il s'agit d'un morphème grammatical, une préposition locative qui vient en tête de syntagme nominal : yeqqim ger Acli d Muhend, «il s'est assis entre Ali et Mohand», mais son origine nominale, comme pour la plupart des prépositions berbères, ne fait aucun doute, comme le confirme sa compatibilité avec les suffixes personnels (= "possessifs»). Le couple chleuh ger/nger-inger recevra la même analyse que précédemment : ger est la forme (anciennement nominale) isolée, alors que la variante nger/inger, s'analyse comme un ancien syntagme $n+$ ger ( $=$ de + entre/milieu/centre).

9 En conséquence, dans cette seconde hypothèse, le Nigris/Gir-Geir pouvait un fleuve « intermediaire », « au milieu de » et « frontière entre » deux ensembles ethniques ou géographiques... La difficulté principale que soulève cette théorie est que la variante nger/inger n'existe que dans le domaine chleuh, et de façon très localisée (notamment Ayt Wawzgit, Ouarzazate, région du Draa), ce qui amène à penser qu'elle est probablement assez récente ; en matière de linguistique historique berbère, vu la rareté des témoignages écrits anciens, il est peu recommandé de se référer à des données locales isolées dont il est quasiment impossible d'établir la pertinence pour des périodes anciennes: seules les faits bien distribués au plan géographique ont des probabilités sérieuses d'être anciens.

10 Ces deux premières analyses postulent que le " $\mathrm{g}$ " des notations antiques correspond bien à un $/ \mathrm{g} /$ en berbère ; or cette correspondance n'est pas absolument certaine, le " $g$ » latin comme le « $\gamma$ » grec, peuvent avoir rendu une autre consonne vélaire libyque qui n'existait pas dans la phonologie des langues classiques, notamment la constrictive vélaire sonore $/ \gamma /$ ( gh » dans les notations courantes du français). Si tel a été le cas, les formes Nigris/Nigritae et Gir/Geir/Giris seraient à mettre en relation avec des

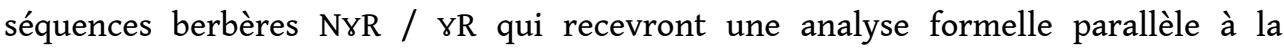
précédente $: N \gamma R=N+\gamma R$. Or, la racine $\gamma R$ est bien attestée sous la forme du thème

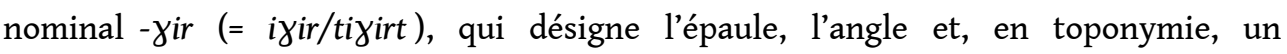
escarpement rocheux. Il existe un cap Ghir au sud d'Agadir et même plusieurs oueds Ghir (ou Rhir), notamment au Sahara, en Petite Kabylie (région de Bougie). Le correspondant berbère serait, dans cette configuration : $n$ (i) yir. 
11 Mais d'autres thèmes nominaux reposent sur racine $\mathrm{YR}$ : - yer / - yar qui renvoient à des significations diverses, dont certaines bien représentées en toponymie et en hydronymie : ayra / tayrat "pierre large et plate»; ayer, "bouclier»; avec de nombreuses formes à redoublement expressif : iyeryer / tiyeryert, "surface plane, plateforme, aire... » (oued Ighargharen, Sahara algérien).

12 En conclusion, quelle que soit la correspondance phonétique berbère que l'on attribue $\mathrm{au}$ « $\mathrm{g} /$ " antique, la seule hypothèse plausible permettant, en berbère, la reconnaissance d'un lien entre les formes Nigris-Nigritae/Geir-Giris... implique une décomposition syntaxique $\mathrm{N}+\mathrm{GR}$ (racine GR) ou $\mathrm{N}+\mathrm{\gamma R}$ (racine $\mathrm{YR}$ ), qui toutes deux présentent une compatibilité sémantique raisonnable avec un usage hydronymique.

13 Mais on ne perdra pas de vue que ces tentatives d'analyses explicatives reposent entièrement sur l'hypothèse de l'existence d'un lien linguistique entre les formes Nigris/Nigritae, d'une part et Gir/Geir/Giris, d'autre part; mais après tout, elles peuvent avoir été totalement étrangères l'une à l'autre et leur rapprochement relever des approximations et identifications, souvent très fantaisistes, des géographes et auteurs anciens.

14 - La recherche des hydronymes nord-africains (Guir, Ghir, Rhir...) a été réalisée sur «Google Earth» (23/04/2011).

INDEX

Mots-clés : Antiquité, Linguistique, Maroc, Onomastique, Sahara, Tribu 